\title{
ON HYPO-JORDAN OPERATORS
}

\author{
EUNGIL KO* \\ Department of Mathematics, Ewha Women's University, Seoul 120-750, Korea \\ e-mail:eiko@mm.ewha.ac.kr
}

(Received 17 January, 2000)

\begin{abstract}
In this paper, we show that if $T=S+N$, where $S$ is similar to a hyponormal operator, $S$ and $N$ commute and $N$ is a nilpotent operator of order $m$ (i.e., $N^{m}=0$ ), then $T$ is a subscalar operator of order $2 m$. As a corollary, we get that such a $T$ has a nontrivial invariant subspace if its spectrum $\sigma(T)$ has the property that there exists some non-empty open set $U$ such that $\sigma(T) \cap U$ is dominating for $U$.
\end{abstract}

1991 Mathematics Subject Classification. 47B20, 47B38.

1. Introduction. Let $H$ be a separable, complex Hilbert space and let $\mathcal{L}(H, K)$ denote the space of all bounded linear operators from $H$ to $K$. If $H=K$, we write $\mathcal{L}(H)$ in place of $\mathcal{L}(H, K)$. Recall that $S \in \mathcal{L}(H)$ is called a hyponormal operator if $S S^{*} \leq S^{*} S$, or equivalently, if $\left\|S^{*} h\right\| \leq\|S h\|$ for every $h \in H$ and $N \in \mathcal{L}(H)$ is called a nilpotent operator of order $m$ if $N^{m}=0$ for some positive integer $m$. An operator $T \in \mathcal{L}(H)$ is said to be hypo-Jordan of order $m$ if $T=S+N$ where $S$ is similar to a hyponormal operator, $S$ and $N$ commute and $N$ is a nilpotent operator of order $m$.

A bounded linear operator $R$ on $H$ is called scalar of order $m$ if it possesses a spectral distribution of order $m$; i.e., if there is a continuous unital morphism of topological algebras

$$
\Phi: C_{0}^{m}(\mathbf{C}) \rightarrow \mathcal{L}(H)
$$

such that $\Phi(z)=R$, where $z$ stands for the identity function on $\mathbf{C}$, and $C_{0}^{m}(\mathbf{C})$ stands for the space of compactly supported functions on $\mathbf{C}$, continuously differentiable of order $m(0 \leq m \leq \infty)$. An operator is subscalar if it is similar to the restriction of a scalar operator to an invariant subspace. As the weaker form of a subscalar operator, we introduce the following: an operator $T \in \mathcal{L}(H)$ is quiasisubscalar if there exists a one-to-one $V \in \mathcal{L}(H, K)$ such that $V T=R V$ where $R(=\Phi(z)$ in the above definition) is a scalar operator. There are examples of quasisubscalar operators in [1].

An operator $T \in \mathcal{L}(H)$ is said to satisfy the single valued extension property if for any open subset $U$ in $\mathbf{C}$, the function

$$
z-T: \mathcal{O}(U, H) \rightarrow \mathcal{O}(U, H)
$$

defined by the obvious pointwise multiplication is one-to-one, where $\mathcal{O}(U, H)$ denotes the space of $H$-valued analytic functions on $U$. If, in addition, the above function $z-T$ has closed range on $\mathcal{O}(U, H)$, then $T$ satisfies Bishop's conditions $(\beta)$.

*The author is supported by the KOSEF, under Grant No. 971-0102-007-2, 1997-99. 
In 1984 M. Putinar proved that any hyponormal operator is subscalar. His theorem was used to show that hyponormal operators with thick spectra have invariant subspaces, a result due to Scott W. Brown [2]. In this paper, we show that if $T=S+N$, where $S$ is similar to a hyponormal operator, $S$ and $N$ commute and $N$ is a nilpotent operator of order $m$, then $T$ is a subscaler operator of order $2 m$. As a corollary, we get that such a $T$ has a nontrivial invariant subspace if its spectrum $\sigma(T)$ has the property that there exists some non-empty open set $U$ such that $\sigma(T) \cap U$ is dominating for $U$.

The paper is organized as follows. In Section 2, we give some preliminary facts. In Section 3, we characterize hypo-Jordan operators and deal with applications of the main result.

2. Preliminaries. Let $z$ be the coordinate in the complex plane $\mathbf{C}$ and $d \mu(z)$ denote the planar Lebesgue measure. Fix a complex (separable) Hilbert space $H$ and a bounded (connected) open subset $U$ of $\mathbf{C}$. We shall denote by $L^{2}(U, H)$ the Hilbert space of measurable functions $f: U \rightarrow H$, such that

$$
\|f\|_{2, U}=\left\{\int_{U}\|f(z)\|^{2} d \mu(z)\right\}^{\frac{1}{2}}<\infty .
$$

The space of functions $f \in L^{2}(U, H)$ that are analytic on $U$ (i.e. $\left.\bar{\partial} f=0\right)$ is denoted by

$$
A^{2}(U, H)=L^{2}(U, H) \cap \mathcal{O}(U, H) .
$$

Then $A^{2}(U, H)$ is called the Bergman space for $U$. It is known that $A^{2}(U, H)$ is complete.

Let us define now a special Sobolev type space. Let $U$ be again a bounded open subset of $\mathbf{C}$ and $m$ a fixed non-negative integer. The vector valued Sobolev space $W^{m}(U, H)$ with respect to $\bar{\partial}$ and of order $m$ will be the space of those functions $f \in L^{2}(U, H)$ whose derivatives $\bar{\partial} f, \cdots, \bar{\partial}^{m} f$ in the sense of distributions still belong to $L^{2}(U, H)$. Endowed with the norm

$$
\|f\|_{W^{m}}^{2}=\sum_{i=0}^{m}\left\|\bar{\partial}^{i} f\right\|_{2, U}^{2}
$$

$W^{m}(U, H)$ becomes a Hilbert space contained continuously in $L^{2}(U, H)$.

Let $U$ be a (connected) bounded open subset of $\mathbf{C}$ and let $m$ be a non-negative integer. The linear operator $M\left(=M_{z}\right)$ of multiplication by $z$ on $W^{m}(U, H)$ is continuous and it has a spectral distribution of order $m$, defined by the functional calculus

$$
\Phi_{M}: C_{0}^{m}(\mathrm{C}) \rightarrow \mathcal{L}\left(W^{m}(U, H)\right), \quad \Phi_{M}(f)=M_{f}
$$

Therefore, $M$ is a scalar operator of order $m$.

3. Main results. In this section, it is shown that any hypo-Jordan operator of order $m$ is subscalar. The starting point of this section deals with the basic inequality for the proof of the main result. 
Lemma 3.1. [8, Proposition 2.1]. For a bounded open disk $D$ in $\mathbf{C}$ there is a constant $C_{D}$ such that, for an arbitrary operator $T \in \mathcal{L}(H)$ and $f \in W^{2}(D, H)$, we have

$$
\|(I-P) f\|_{2, D} \leq C_{D}\left(\left\|(z I-T)^{*} \bar{\partial} f\right\|_{2, D}+\left\|(z I-T)^{*} \bar{\partial}^{2} f\right\|_{2, D}\right),
$$

where $P$ denotes the orthogonal projection of $L^{2}(D, H)$ onto the Bergman space $A^{2}(D, H)$.

COROllary 3.2. [8, Corollary 2.2]. If $S$ is hyponormal, then

$$
\|(I-P) f\|_{2, D} \leq C_{D}\left(\|(z-S) \bar{\partial} f\|_{2, D}+\left\|(z-S) \bar{\partial}^{2} f\right\|_{2, D}\right),
$$

where z denotes $z I$.

Lemma 3.3. Let $T \in \mathcal{L}(H)$ be an operator such that $T=S+N$, where $S$ is similar to a hyponormal operator, $S$ and $N$ commute and $N$ is a nilpotent operator of order $m$. Let $D$ be a bounded disk which contains $\sigma(T)$. Then the operator $V: H \rightarrow H(D)$, defined by $V h=1 \otimes h+\overline{(z-T) W^{2 m}(D, H)}(=1 \widetilde{\otimes})$, is one-to-one and has closed range, where $H(D)=W^{2 m}(D, H) / \overline{(z-T) W^{2 m}(D, H)}$ for $m=1,2, \cdots$ and $1 \otimes h$ denotes the constant function sending any $z \in D$ to $h$.

Proof. It suffices to prove the following assertion: if $h_{n} \in H$ and $f_{n} \in W^{2 m}(D, H)$ are sequences such that

$$
\lim _{n \rightarrow \infty}\left\|(z-T) f_{n}+1 \otimes h_{n}\right\|_{W^{2 m}}=0
$$

then $\lim _{n \rightarrow \infty} h_{n}=0$.

By the definition of the norm a Sobolev space, the assertion (1) implies that

$$
\lim _{n \rightarrow \infty}\left\|(z-T) \bar{\partial}^{i} f_{n}\right\|_{2, D}=0
$$

for $i=1,2, \cdots, 2 m$. Since $T=S+N$, we have

$$
\lim _{n \rightarrow \infty}\left\|(z-S) \bar{\partial}^{i} f_{n}-N \bar{\partial}^{i} f_{n}\right\|_{2, D}=0
$$

for $i=1,2, \cdots, 2 m$. From the equation (3) and $S N=N S$, we have

$$
\lim _{n \rightarrow \infty}\left\|(z-S) \bar{\partial}^{i}\left(N^{k} f_{n}\right)-N^{k+1} \bar{\partial}^{i} f_{n}\right\|_{2, D}=0
$$

for $i=1,2, \cdots, 2 m$ and $k=0,1, \cdots, m-1$. If in particular $k=m-1$, then

$$
\lim _{n \rightarrow \infty}\left\|(z-S) \bar{\partial}^{i}\left(N^{m-1} f_{n}\right)\right\|_{2, D}=0
$$

for $i=1,2, \cdots, 2 m$.

Claim. $\lim _{n \rightarrow \infty}\left\|(z-S) \bar{\partial}^{i}\left(N^{m-j} f_{n}\right)\right\|_{2, D}=0 \quad$ for $\quad i=1,2, \cdots, 2(m+1-j)$ and $j=1,2, \cdots, m$.

We prove this claim by induction. If $j=1$, it is clear from the equation (5). We assume that the above claim holds for some given $j=1,2, \cdots, m-1$. Indeed, 


$$
\lim _{n \rightarrow \infty}\left\|(z-S) \bar{\partial}^{i}\left(N^{m-j} f_{n}\right)\right\|_{2, D}=0
$$

for $i=1,2, \cdots, 2(m+1-j)$ and $j=1,2, \cdots, m-1$.

We only need to verify that

$$
\lim _{n \rightarrow \infty}\left\|(z-S) \bar{\partial}^{i}\left(N^{m-(j+1)} f_{n}\right)\right\|_{2, D}=0
$$

for $i=1,2, \cdots, 2(m+1-(j+1))$ and $j=1,2, \cdots, m-1$.

Since $S$ is similar to a hyponormal operator $B$, there exists an invertible operator $R$ such that $R S=B R$. From the equation (6) we have

$$
\lim _{n \rightarrow \infty}\left\|R(z-S) \bar{\partial}^{i}\left(N^{m-j} f_{n}\right)\right\|_{2, D}=0
$$

for $i=1,2, \cdots, 2(m+1-j)$ and $j=1,2, \cdots, m-1$. From the equation (7) and $R S=B R$ we get

$$
\lim _{n \rightarrow \infty} \|(z-B) R \bar{\partial}^{i}\left(N^{m-j} f_{n} \|_{2, D}=0\right.
$$

for $i=1,2, \cdots, 2(m+1-j)$ and $j=1,2, \cdots, m-1$. By Corollary 3.2,

$$
\left\|(I-P) \bar{\partial}^{i}\left(R N^{m-j} f_{n}\right)\right\|_{2, D} \leq C_{D}\left(\left\|(z-B) \bar{\partial}^{i+1}\left(R N^{m-j} f_{n}\right)\right\|_{2, D}+\left\|(z-B) \bar{\partial}^{i+2}\left(R N^{m-j} f_{n}\right)\right\|_{2, D}\right)
$$

for $i=1,2, \cdots, 2(m+1-(j+1))$ and $j=1,2, \cdots, m-1$. From the equation (8),

$$
\lim _{n \rightarrow \infty}\left\|(I-P) \bar{\partial}^{i}\left(R N^{m-j} f_{n}\right)\right\|_{2, D}=0
$$

for $i=1,2, \cdots, 2(m+1-(j+1))$ and $j=1,2, \cdots, m-1$. By the equations (8) and (9) we see that

$$
\lim _{n \rightarrow \infty}\left\|(z-B) P\left[\bar{\partial}^{i}\left(R N^{m-j} f_{n}\right)\right]\right\|_{2, D}=0
$$

for $i=1,2, \cdots, 2(m+1-(j+1))$ and $j=1,2, \cdots, m-1$. Since every hyponormal operator has the property $(\beta)$ (see [7, Theorem 5.5]), we get that for $i=1,2, \cdots, 2(m+1-(j+1))$ and $j=1,2, \cdots, m-1$,

$$
P\left(\bar{\partial}^{i} R N^{m-j} f_{n}\right) \rightarrow 0
$$

uniformly on compact subsets of $D$. Therefore, it is easy to show that

$$
\lim _{n \rightarrow \infty}\left\|P\left[\bar{\partial}^{i}\left(R N^{m-j} f_{n}\right)\right]\right\|_{2, D}=0
$$


for $i=1,2, \cdots, 2(m+1-(j+1))$ and $j=1,2, \cdots, m-1$. From the equations (9) and (11), we have

$$
\lim _{n \rightarrow \infty}\left\|\bar{\partial}^{i}\left(R N^{m-j} f_{n}\right)\right\|_{2, D}=0
$$

for $i=1,2, \cdots, 2(m+1-(j+1))$ and $j=1,2, \cdots, m-1$. Since $R$ is invertible, we get from (12) that

$$
\lim _{n \rightarrow \infty}\left\|\bar{\partial}^{i}\left(N^{m-j} f_{n}\right)\right\|_{2, D}=0
$$

for $i=1,2, \cdots, 2(m+1-(j+1))$ and $j=1,2, \cdots, m-1$. From the equations (4) and (13),

$$
\lim _{n \rightarrow \infty}\left\|(z-S) \bar{\partial}^{i}\left(N^{m-(j+1)} f_{n}\right)\right\|_{2, D}=0
$$

for $i=1,2, \cdots, 2(m+1-(j+1))$ and $j=1,2, \cdots, m-1$, and so this completes the proof of the claim stated above.

Let us come back now to the proof of Lemma 3.3. By the claim, the following equation holds:

$$
\lim _{n \rightarrow \infty}\left\|(z-S) \bar{\partial}^{i} f_{n}\right\|_{2, D}=0
$$

for $i=1,2$. Since $R$ is bounded,

$$
\lim _{n \rightarrow \infty}\left\|R(z-S) \bar{\partial}^{i} f_{n}\right\|_{2, D}=0
$$

for $i=1,2$. Since $R S=B R$, from the equation (15) we have

$$
\lim _{n \rightarrow \infty}\left\|(z-B) \bar{\partial}^{i}\left(R f_{n}\right)\right\|_{2, D}=0
$$

for $i=1,2$. By Corollary 3.2 and the equation (16), we get

$$
\lim _{n \rightarrow \infty}\left\|(I-P) R f_{n}\right\|_{2, D}=0 .
$$

Set $g_{n}=R^{-1} P\left[R f_{n}\right]$. The $g_{n} \in A^{2}(D, H)$. Since

$$
\left\|f_{n}-g_{n}\right\|_{2, D} \leq\left\|R^{-1}\right\|\left\|R f_{n}-P\left[R f_{n}\right]\right\|_{2, D},
$$

the equation (17) implies that

$$
\lim _{n \rightarrow \infty}\left\|f_{n}-g_{n}\right\|_{2, D}=0
$$


Now from (1) and (18) we obtain the following equation.

$$
\lim _{n \rightarrow \infty}\left\|(z-T) g_{n}+1 \otimes h_{n}\right\|_{2, D}=0 .
$$

Let $\Gamma$ be in a circle in $D$ surrounding $\sigma(T)$. Then for $z \in \Gamma$

$$
\lim _{n \rightarrow \infty}\left\|g_{n}(z)-(z-T)^{-1}\left(1 \otimes h_{n}\right)\right\|=0
$$

uniformly. Hence, by the Riesz functional calculus,

$$
\lim _{n \rightarrow \infty}\left\|\frac{1}{2 \pi i} \int_{\Gamma} g_{n}(z) d z+h_{n}\right\|=0,
$$

where it is assumed that $\Gamma$ is described once counterclockwise.

But $\int_{\Gamma} g_{n}(z) d z=0$ by Cauchy's theorem. Hence $\lim _{n \rightarrow \infty} h_{n}=0$. Thus $V$ is oneto-one and has closed range.

Now we state and prove the main theorem.

THEOREM 3.4. If $T$ is any operator such that $T=S+N$, where $S$ is similar to a hyponormal operator, $S$ and $N$ commute and $N$ is a nilpotent operator of order $m$ (i.e. $T$ is any hypo-Jordan operator of order $m$ ), then $T$ is a subscalar operator of order $2 m$.

Proof. Consider an arbitrary bounded open disk $D$ in the complex plane $\mathbf{C}$ that contains $\sigma(T)$ and the quotient space

$$
H(D)=W^{2 m}(D, H) / \overline{(z-T) W^{2 m}(D, H)}
$$

endowed with the Hilbert space norm. Let $M\left(=M_{z}\right)$ be the operator of multiplication by $z$ on $W^{2 m}(D, H)$. Then $M$ is a scalar operator of order $2 m$ and its spectral distribution is given by

$$
\Phi: C_{0}^{2 m}(\mathrm{C}) \rightarrow \mathcal{L}\left(W^{2 m}(D, H)\right), \quad \Phi(f)=M_{f},
$$

where $M_{f}$ is the operator of multiplication by $f$. Since $M$ commutes with $z-T, \tilde{M}$ on $H(D)$ is still a scalar operator of order $2 m$, with $\tilde{\Phi}$ as a spectral distribution.

Let $V$ be the operator

$$
V h=1 \otimes h+\overline{(z-T) W^{2 m}(D, H)} \quad(=1 \otimes h),
$$

from $H$ into $H(D)$, denoting by $1 \otimes h$ the constant function $h$. Then $V T=\tilde{M} V$. By Lemma 3.3, $V$ is one-to-one and has closed range. Therefore, ran $V$ is a closed invariant subspace for the scalar operator $\tilde{M}$. Hence $T$ is a subscalar operator of order $2 m$.

Recall that if $U$ is a non-empty open set in $\mathbf{C}$ and if $\Omega \subset U$ has the property that 


$$
\sup _{\lambda \in \Omega}|f(\lambda)|=\sup _{\beta \in U}|f(\beta)|
$$

for every function $f$ in $H^{\infty}(U)$ (i.e. for all $f$ bounded and analytic on $U$ ), then $\Omega$ is said to be dominating for $U$.

COROLlary 3.5. Let $T \in \mathcal{L}(H)$ be any hypo-Jordan operator of order $m$. If $\sigma(T)$ has the property that there exists some non-empty open set $U$ such that $\sigma(T) \cap U$ is dominating for $U$, then $T$ has a nontrivial invariant subspace.

Proof. The proof follows that Theorem 3.4 and [4].

COROLlary 3.6. Any hypo-Jordan operator has the property $(\beta)$.

Proof. Since every scalar operator has the property $(\beta)$ (see $[\mathbf{8}]$ ) and the property $(\beta)$ is transmitted from an operator to its restrictions to closed invariant subspaces, it follows from Theorem 3.4 that any hypo-Jordan operator has the property $(\beta)$.

COROLlary 3.7. If $T=S+N$ is hypo-Jordan of order $m$ and quasinilpotent, then $T$ is a nilpotent operator of order $m$.

Proof. Since $\sigma(S)=\sigma(T)=\{0\}$, an operator $S$ is quasinilpotent and is similar to a hyponormal operator. Therefore, $S$ is a zero operator. Hence $T=N$.

Recall that an operator $X \in \mathcal{L}(H, K)$ is called a quasiaffinity if it has trivial kernel and dense range. An operator $A \in \mathcal{L}(H)$ is said to be a quasiaffine transform of an operator $T \in \mathcal{L}(K)$ if there is a quasiaffinity $X \in \mathcal{L}(H, K)$ such that $X A=T X$.

Corollary 3.8. Let $T \in \mathcal{L}(H)$ be any hypo-Jordan operator. If $A$ is any quasiaffine transform of $T$, then $\sigma(T) \subset \sigma(A)$.

Proof. The proof follows from Corollary 3.6 and [6, Theorem 3.2].

Corollary 3.9. Let $T \in \mathcal{L}(H)$ be any hypo-Jordan operator. If $A$ is any quasiaffine transform of $T$, then $A$ is quasisubscalar.

Proof. Let $X \in \mathcal{L}(H, K)$ be a quasiaffinity such that $X A=T X$. Since $V$ (in Theorem 3.4) and $X$ are one-to-one, $V X$ is one-to-one. Therefore, $V X$ implements the quasisubscalar properties. Thus $A$ is quasisubscalar.

In the following theorem we establishes an analogue of the single valued extension property for the space $W^{k}(D, H)$.

Proposition 3.10. If $T \in \mathcal{L}(H)$ is a hypo-Jordan operator of order $m$, then the operator

$$
z-T: W^{2 m}(D, H) \rightarrow W^{2 m}(D, H)
$$

is one-to-one, for an arbitrary bounded disk $D$ in $\mathbf{C}$. 
Proof. Let $f \in W^{2 m}(D, H)$ be such that $(z-T) f=0$. Then by a similar method as in the proof of Lemma 3.3, we can show that $R f=P R f \in A^{2}(D, H)$ (c.f. (17)). Since $T$ is subscalar, by Theorem 3.4, we know that $T$ has the single valued extension property. Therefore, $P R f=0$; i.e., $f=0$. Thus $z-T$ is one-to-one.

Corollary 3.11. Let $T=S+N$ be such that $S N=N S$, where $S$ is similar to a normal operator and $N$ is quasinilpotent. Let $\sigma(T)$ lie in a $C^{1}$-Jordan curve. Suppose that there exists a constant $M$ such that

$$
\left\|(z-T)^{-1}\right\| \leq M /\{\operatorname{dist}(z, \sigma(T))\}^{m}
$$

for all $z \in \rho(T)$ with $|z| \leq\|T\|+1$. Then $T$ is subscalar of order $2(4 m+4)$.

Proof. We know that $N^{4 m+4}=0$ by [9, Corollary 1.10] and so it follows from Theorem 3.4 that $T$ is subscalar of order $2(4 m+4)$.

\section{REFERENCES}

1. S. W. Brown and E. Ko, Operators of Putinar type, Operator theory: Adv. Appl. 104 (Birkhäuser-Verlag, 1998).

2. S. W. Brown, Hyponormal operators with thick spectra have invariant subspaces, Ann of Math. 125 (1987), 93-103.

3. J. Eschmeier and M. Putinar, Bishop's condition $(\beta)$ and rich extensions of linear operators, Indiana Univ,. Math. J. 37 (1988), 325-348.

4. J. Eschmeier, Invariant subspaces for subscalar operators. Arch. Math. (Basel) 52 (1989), 562-570.

5. E. Ko, Algebraic and triangular n-hyponormal operators, Proc. Amer. Math. Soc. 123 (1995), 3473-3481.

6. E. Ko, On a Clary theorem, Bull. Korean Math. Soc. 33 (1996), 29-33.

7. M. Martin and M. Putinar, Lectures on hyponormal operators, Operator Theory: Adv Appl. 39 (Birkhäuser-Verlag, 1989). 395.

8. M. Putinar. Hyponormal operators are subscalar, J. Operator Theory 12 (1984), 385-

9. B. L. Wadhwa. Spectral, $M$-hyponormal and decomposable operators, Ph.D. thesis, Indiana University, 1971. 